\title{
THE MODERNIZATION OF THE PULKOVO PHOTOGRAPHIC (PHOTOELECTRIC) VERTICAL CIRCLE BY A CCD ARRAY
}

\author{
G. A. Goncharov, B. K. Bagildinsky, E. V. Kornilov, D. D. Polojentsev, \\ K. V. Rumyantsev and V. D. Shkutov \\ Pulkovo Observatory
}

The Zverev photographic vertical circle (PVC) of the Pulkovo observatory is in the process of modernization. The features of the vertical circle are:

a) Maksutov mirror-lens optical system with small aberrations and wide passband: aperture: $20 \mathrm{~cm}$, focal length: $200 \mathrm{~cm}$, focal scale: $103 \mathrm{arcsec} / \mathrm{mm}$.

b) Very compact instrument: $140 \mathrm{~cm}$ total length, $60 \mathrm{~cm}$ - tube.

c) Wide field: $25 \times 25 \mathrm{~mm}=40^{\prime} \mathrm{X} 40^{\prime}$. Wide-field imaging can be combined with meridian observations.

d) Easily-reversible instrument: reversing takes less than 30 seconds.

e) Two divided vertical circles of glass. Photoelectric circle reading microscopes.

f) Photographic micrometer in focal plane. This will be changed with a CCD micrometer.

The Photoelectric Vertical Circle makes possible, as do usual vertical circles, the determination of stellar declinations in an absolute manner. The observed zenith distance is a linear combination of micrometer, circle and level readings before and after the reversing of the instrument. Therefore all zero-points are eliminated and the final value of the zenith distance is free from variations of the instrumental system orientation between observations.

There are three versions of the new CCD micrometer for the Photoelectric Vertical Circle: a) An array of three CCDs arrays, $1040 \times 1160$ pixels, pixel size of $16 \times 16 \mu \mathrm{m}$, covers a field $27 \times 31$ arcmin. In this case two arrays with filters register in two bands from $U$ to I and a third array registers in a wide passband.

b) An array of two such CCDs in which the first is covered by two filters and the second works in a wide passband.

c) One array registers all bands with changed filters.

The CCD array allows us simultaneously to observe in the three bands (during one passage of an object). With one CCD we have to make the observations during two passages (nights). 
We intend to observe ALL stars and other compact objects (extragalactic, asteroids, planet satellites and artificial satellites) from $7^{\text {th }}$ to $18^{\text {th }}$, up to the pole in declination.

The aims are:

a) Absolute and differential determination of both coordinates will be made by classic and/or modern methods which use CCD abilities (Hoeg 1995, Goncharov 1995). A determination will be made directly with respect to an extragalactic reference frame. A link of Hipparcos/Tycho stars with quasars is possible.

b) Photometric determinations will be made of color indices for the purpose of elimination of chromatic refraction and for astrophysical studies.

The expected accuracy of the differential coordinate determinations is 50 mas and of the photometric determinations is $0.01-0.1$ magnitude. A special light mark near the array and equipment on the tube will allow us to control instantly the flexure of the tube. Improvements in accuracy are expected by means of network of environmental and instrumental sensors of temperature, pressure, humidity, currents, voltages and signals.

\section{TABLE 1}

Some results of the PVC's modernization.

\begin{tabular}{lrr}
\hline \hline & Current PVC & Upgraded PVC \\
\hline Observed Quantity & DECLINATION & BOTH COORDINATES \\
& AND PHOTOMETRY \\
\hline MAGNITUDE LIMIT & 8 & 18 \\
ACCURACY, MAS & 180 & 50 \\
OBJECTS IN FRAME & 1 & 1500 \\
OBJECTS: TOTAL & 2000 & $300,000,000$ \\
OBSERVATIONS/YEAR & 3000 & $70,000,000$ \\
\hline
\end{tabular}

\section{ACKNOWLEDGEMENT}

This research was carried out with financial support from the Russian Foundation of Fundamental Research, grant \# 93-02-3056.

\section{REFERENCES}

Hoeg, E. 1995 in IAU Symposium No. 166, Astronomical and Astrophysical Objectives, Kluewer Academic Pub., in press

Goncharov, G. 1995 in IAU Symposium No. 166, Astronomical and Astrophysical Objectives of Sub-Millisecond Optical Astrometry, Kluwer Academic Pub., Dordrecht, in press 